\title{
Las elecciones presidenciales en Brasil [2018): La cobertura de la prensa de referencia argentina
}

\section{Marina Acosta' Silvia Demirdjian²}

\section{Resumen}

Los procesos electorales en general representan una interesante oportunidad para analizar el posicionamiento de la prensa. Los medios de comunicación tematizan esas coyunturas y en su construcción discursiva ofrecen ciertas representaciones/encuadres de los panoramas sociales, económicos y políticos de los países donde tienen lugar las elecciones que narran y comentan.El objetivo general de la investigación es analizar comparativamente la cobertura de la prensa de referencia argentina sobre el triunfo de Jair Bolsonaro. El estudio describe la agenda que han activado los dos periódicos de mayor circulación en Argentina, Clarín y La Nación, durante octubre de 2018. La metodología utilizada es el clásico análisis de contenido; para asistiral análisis del proceso exploratorio se utiliza también el software informático ATLAS/ $t i$. El marco teórico se sustenta en la teoría de la agenda-setting, la tematización y el proceso de enmarcado noticioso (framing). Dentro de los principales hallazgos de la investigación destacan que el índice de importancia de la cobertura ha oscilado entre el rango medio-alto y máximo, que el temario sobre el proceso electoral brasileño se concentró en las noticias que tenían como protagonista a Bolsonaro, que ambos periódicos utilizaron información propia en la construcción discursiva de los comicios y que el encuadre de campaña que se impuso fue el estratégico.

\section{Palabras-clave}

Agenda-Setting; Framing; Elecciones; Prensa De Referencia; Brasil; Argentina. 


\section{The presidential elections in Brazil [2018): The coverage of the Argentine reference press}

\section{Marina Acosta' Silvia Demirdjian²}

\section{Abstract}

The electoral processes in general represent an interesting opportunity to analyze the positioning of the press. The media thematize these situations and in their discursive construction they offer certain representations/frames of the social, economic and political scenarios of the countries where the elections that narrate and comment take place. The general objective of the investigation is to analyze comparatively the coverage of the Argentine reference press on the triumph of Jair Bolsonaro. The study describes the agenda that has been activated by the two news papers with the highest circulation in Argentina, Clarín and La Nación, during October 2018. The methodology used is the classic content analysis. To assist in the analysis of the exploratory process, the ATLAS.ti software is also used. The theoretical framework is based on the theory of agenda-setting, thematization and framing. Among the main findings of the investigation: the coverage importance index has ranged between the medium-high and maximum range, the agenda on the Brazilian electoral process focused on the news that had Bolsonaro as its protagonist, both newspapers used their own information in the discursive construction of the elections and the main frame was the strategic.

\section{Keywords}

Agenda-Setting; Framing; Elections; Reference press; Brasil; Argentina 


\section{Introducción}

Brasil y Argentina mantienen una histórica relación y han sido punta de lanza en la profundización de los lazos de integración regional y comercial. Múltiples estudios han dado cuenta del interés que para Argentina representa la vecina economía(HIRST, 1990; RUSSELL; TOKATLIAN, 2003). Asimismo, respecto de su posicionamiento en tema de política exterior ambos países han atravesado un similar giro discursivo hacia la izquierda con el ascenso del lulopetismo y el kirchnerismo al poder (GAGLIARDI, 2018; KEJSEFMAN, 2017).

Durante el transcurso de 2018, México y Brasil (las dos principales economías de la región) llevaron adelante sus respectivos calendarios de votación presidencial. Antes de la realización de los comicios en el país azteca reinaba la incertidumbre: el ocaso del priismo podría permitir, por primera vez en la historia, la llegada de la izquierda al gobierno de la mano de Andrés Manuel López Obrador. En Brasil, con Lula encarcelado, el triunfo de Jair Bolsonaro abrió una nueva etapa que pautó la llegada al poder de la ultraderecha.

Los procesos electorales de América Latina, en general, constituyen en sí un criterio de noticiabilidad para la prensa. En este sentido, representan una interesante oportunidad para analizar las estrategias editoriales llevadas adelante por los media. En rigor, los medios miran necesariamente esas coyunturas y en su construcción discursiva ofrecen ciertas representaciones de los panoramas sociales, económicos y políticos de los países donde tienen lugar las elecciones que narran y comentan.

A esto hay que sumar que los comicios representan momentos de generación de agenda política y de debate mediático acerca de las propuestas de los candidatos y los partidos políticos (MUÑIZ; ROMERO, 2012). De allí el interés por conocer cuál es la tematización y el proceso de encuadre noticioso (framing) que la prensa de referencia argentina ha producido sobre las elecciones presidenciales en Brasil.

Este estudio describe la agenda que han activado los dos periódicos de mayor circulación en Argentina- Clarín y La Nación- durante octubre de 2018. El interés de este trabajo, en términos generales, se funda en la necesidad de conocer cómo la prensa argentina agenda la realidad electoral de los países latinoamericanos. De cara a los complejos procesos que enfrenta la economía global, Brasil en particular es un caso interesante de análisis por un doble motivo. En primer lugar, porque representa la primera economía de América Latina y, en segundo lugar, porque tras los resultados de 2018 se inauguró un nuevo ciclo histórico- como observáramos ut supra- en sintonía con el ascenso de la derecha en la región. 
Se pueden relevar múltiples trabajos de la academia brasileña sobre la cobertura mediática que su prensa de referencia ha realizado sobre diversos procesos electorales al interior de sus fronteras nacionales (GAGLIARDI, 2018). No obstante, queda vacante el estudio sobre la ponderación que los periódicos de sendos países otorgan a este tipo de acontecimientos políticos (campañas y elecciones presidenciales) en Brasil.

Pocos estudios versan sobre el tema; tal es el caso del trabajo de Maurente Laflor (2016) sobre la mirada de la prensa argentina a la participación de las candidaturas presidenciales brasileñas durante el proceso electoral de 2010. Así, resulta paradójico el hecho de que sean pocos los trabajos específicos desarrollados en esta perspectiva, en tanto todo resultado comicial reporta consecuencias de relevancia en el vinculo binacional.

\section{Marco Teórico}

Desde el punto de vista de las teorías del periodismo hubo un intenso debate concentrado en explicar el pasaje de una concepción que creía que los medios representaban la realidad a una que advierte que la construyen. Por lo que las nuevas visiones demostraron que los media no son simples espejos de la realidad sino fundamentalmente constructores de realidad social y, también, poderosos actores políticos (BORRAT, 1989; RODRIGO ALSINA, 1989; BORRAT; DE FONTCUBERTA, 2006). Además, gozan del poder de informar; es decir de una situación de dominio intelectual capaz de influir en personas e instituciones (NIETO; IGLESIAS, 1993).

\section{Agenda-setting}

Maxwell McCombs y Donald Shaw definen a la fijación de agenda (agenda-setting function) como "la capacidad de losmediosmasivos de seleccionar y destacar ciertos temas sobre otros, y con ello causar que los asuntos destacados sean percibidos como importantes por el público" (1977, p. 12). Sin ese conjunto de problemas a resolver, que además está ordenado por prioridades, la funcionalidad de la sociedad como tal sería insostenible (LÓPEZ-ESCOBAR et al., 1996).

La hipótesis de la agenda-setting conlleva dos ideas interrelacionadas: a) la prensa gráfica presenta al público un conjunto de temas sobre los que pensar a través de un proceso de selección y jerarquización informativa (primer nivel); b) la prensa gráfica ofrece un conjunto de atributos sustantivos que influyen de manera decisiva en la interpretación del acontecimiento noticioso (segundo nivel).

José Luis Dader propone la expresión efecto de canalización para referirse a 
la orientación o conducción que "las mentes de los ciudadanos sufren hacia unos repertorios de temas de preocupación pública, en detrimento de otros que no son mencionados o destacados, como consecuencia de la elección previa de asuntos que realizan los mass media" (1992, p. 295). Por cierto, en la acción de los medios están implícitos los sesgos que determinan tal canalización y que dependen de las rutinas productivas de los profesionales que forman parte de ellos.

El proceso de la agenda-setting se compone de los tres actores (y sus interrelaciones) que están involucrados en la comunicación política; esto es, los políticos, los medios de comunicación y los ciudadanos. Se trata, en definitiva, de una perspectiva que concibe a la agenda-setting como un modelo complejo de relaciones entre distintos actores sociales y políticos, que la lleva a alejarse del enfoque estrictamente centrado en la mera transferencia temática del mensaje (ROGERS; DEARING, 1988).

El contenido de la agenda, a su vez, está integrado por tres niveles: 1) composición: referida a la importancia del tema; 2) enfoque: referido a su definición; 3) evaluación/valoración: referida a la instauración de un clima de opinión (NOELLENEUMANN; MATHES, 1987).

\section{Framing}

Una rama de los estudios que intenta echar luz sobre las estrategias que los medios impresos ponen en circulación en su tarea diaria de contar la realidad se centra en los encuadres; es decir, enlasideas vectoras que organizan el relato periodístico (Tankard et al., 1991). A este tipo de estudio se los conoce como framing que, desde nuestra visión, constituye una superación a la teoría de la agenda-setting (MCCOMBS \& SHAW, 1972; SAUTU, 2005) en tanto puede ser definido como una teoría integral que permite articular las distintas etapas de la comunicación (SÁDABA, 2008) además de constituir un proceso dinámico e interactivo dentro del constructivismo social (ARUGUETE, 2011; 2015; 2017).

Preocupado por estudiar cómo los medios construyen encuadres, el framing pasa a ser un proceso de transmisión de sentido que va de los medios a la audiencia y de la audiencia a los medios (AMADEO, 2002). Para Teresa Sádaba, los periodistas otorgan a las noticias un enfoque o idea central que aporta un contexto mediante un proceso de selección, énfasis, y elaboración que influye de manera decisiva en la interpretación del hecho noticioso (2008).

El framing define a los asuntos y proporciona el marco interpretativo para comprenderlo, produciendo y limitando el significado de los issues. Para hacerlo, los 
medios definen una estrategia que se sustenta en un doble principio: el de la elección temática y el de la organización discursiva (MATTHES, 2012).

En la cobertura de los asuntos políticos, predomina el encuadre de conflicto (DE VREESE, 2014; MUÑIZ, 2015; MUÑIZ et al., 2018). Se trata de un encuadre noticioso generalista que se utiliza en cualquier suceso noticiable para enfatizar el desacuerdo entre distintos actores involucrados en políticas (SEMETKO \& VALKENBUG, 2000).

La disputa entre ellos aumenta la probabilidad de impacto de la información con la consecuente canalización cognitiva que configura opinión es del público (MUÑIZ et al., 2018).

\section{Tematización}

El sistema político es una fuente inagotable de noticias (RODRIGO ALSINA, 1989). Al mismo tiempo, son los acontecimientos políticos los que diariamente organizan los titulares de los periódicos. En este sentido, la tematización representa una corriente teórica que nos permite analizar las relaciones entre los medios de comunicación y la política.

La organización de la realidad que propone el sistema político y que se transforma en tematización por la acción de los medios de comunicación activa una no sólo una agenda de opinión sino además un proceso de discusión pública. Por cierto, la principal función de los medios es procesar temas sobre los que se tomen posición (LUHMANN, 2000). Particularmente, los temas políticos activan un sistema de flujos de argumentación tendiente a que la opinión pública se posicione frente a la discusión que despierta.

La tematización se define como "el proceso de definición, establecimiento y reconocimiento público de los grandes temas, los grandes problemas políticos que constituyen la opinión pública, mediante la acción determinante de los medios de comunicación" (SAPERAS, 1987, p. 92). Se trata de un proceso tripartito a partir de la relación establecida entre el sistema de medios, la opinión pública y el sistema político.

La aplicación empírica de esta teoría permite analizar el discurso periodístico de la prensa de referencia para reconocer sus estrategias argumentativas y sus posicionamientos políticos respecto de un tema (GROSSI, 1992). Asimismo, la producción de opinión de un tema político muestra a los participantes sociales que intervienen en su definición y discusión (SANTILLÁN, 2012). 
El objetivo general de la investigación es analizar comparativamente la cobertura de la prensa de referencia argentina sobre los comicios brasileños. Son objetivos específicos: 1) determinar el "índice de relevancia informativa" sobre el proceso electoral; 2) identificar los usos de los encuadres de campaña; 3) precisar las fuentes utilizadas en la construcción del relato periodístico; 4) explicitar, desde los titulares, los encuadres cognitivos.

Para cumplir las metas trazadas, recurrimos al clásico análisis de contenido(AC). Se trata de una metodología destinada a formular, a partir de ciertos datos, inferencias reproducibles y válidas que puedan aplicarse a un contexto (KRIPPENDORFF, 1990, p. 28). Al tratarse de un conjunto de procedimientos interpretativos basados en técnicas de medida cuantitativas (estadísticas basadas en el recuento de unidades) y cualitativas (lógicas basadas en la combinación de categorías) (PIÑUEL, 2002), creemos que el AC es el método más apropiado para responder a los objetivos de la investigación.

El AG, con categorías que se detallan más adelante, se complementa con el análisis de framing de las unidades informativas que componen la muestra de análisis. Tal propuesta se enmarca en la idea de que: a) los medios aportan un contexto mediante un proceso de selección, énfasis, exclusión y elaboración (SÁDABA, 2008) a través de la construcción de marcos interpretativos o frames (ideas vectoras, centrales u organizadoras del discurso) para narrar y comentar la realidad ; b) las agendas de los medios constituyen lugares para registrar hechos y observar/analizar los posicionamientos de quienes han sido actores directos de los distintos acontecimientos relacionados con la temática en cuestión, c) los encuadres destacados suscitan respuestas cognitivas congruentes en los sujetos que las procesan, "influyendo en la formación de opiniones hacia ciertos grupos o colectivos sociales" (IGARTÚA et al., 2007, p. 95).

Para asistir al análisis del proceso exploratorio y dar cuenta de la tematización también utilizamos el software informático ATLAS/ti. El mismo nos permitió: 1) recopilar y clasificar datos en una misma unidad hermenéutica; en nuestro caso compuesto por cada una de las unidades informativas que componen la muestra; 2) realizar redes semánticas que posibilitaron la comprensión global del discurso de la prensa de referencia.

\section{Los medios analizados}

La muestra está compuesta por dos periódicos "generalistas" de circulación 
nacional: Clarín y La Nación. La elección de los medios no ha sido aleatoria. Por el contrario, se los ha incluido porque representan a la prensa de referencia argentina; es decir, porque son los periódicos de mayor tirada a nivel nacional (Clarín con un promedio mensual de 206 mil ejemplares y La Nación con un promedio mensual de 103 mil ejemplares [1] y su tematización impacta en las agendas de la opinión pública y en la de medios más pequeños. Además, existe una influencia recíproca entre ambos (CASERMEIRO, 2004).

Clarín fundado, en 1945, por el periodista y estanciero Roberto Noble. Apareció con un innovador formato tabloide y con una moderna diagramación, inspirado en el inglés The Mirror. Es el diario de mayor tirada de Argentina, según el IVC. Actualmente cuenta con más de cien mil suscriptores digitales, a través del sistema paywall, que le permite a los lectores acceder al contenido impreso que el periódico publica diariamente [2]. Forma parte del poderoso multimedio Grupo Clarín [3].

La Nación fue fundado en 1870 por Bartolomé Mitre, expresidente, general del Ejército y por entonces senador de la Nación, bajo el lema La Nación será una tribuna de doctrina. El formato que lo caracterizó hasta hace poco era el sábana; hoy ha virado hacia el tabloide. Cuando Mitre fundó el diario pretendía tener una publicación que contribuyera a consolidar la organización nacional (ULANOVSKY, 2005). En efecto, su preocupación por recordar a los distintos gobiernos el contenido de los preceptos constitucionales se enmarcó en la defensa del orden social (SIDICARO, 1989).

Desde la perspectiva de Steimberg y Traversa (1997), Clarín es un agente de información, en tanto La Nación es un conductor de opinión. Por tanto, nos encontramos con dos medios que sustentan la función de la prensa escrita: la narración y el comentario (BORRAT, 1998).

\section{Muestra y universo de análisis}

La muestra se obtuvo mediante las hemerotecas de las réplicas digitales de las ediciones impresas de Clarín y La Nación. El universo de análisis comprende las unidades informativas publicadas en el período que comprende desde el 8 de octubre de 2018 (edición que registra los resultados de la primera vuelta) al 29 de octubre de 2018 (edición que registra los resultados de la segunda vuelta).

La ficha técnica del trabajo de campo se compone de la siguiente manera:

1) Ámbito: elecciones presidenciales en Brasil; 2) Universo: totalidad de la información publicada sobre las elecciones en Brasil en las secciones El Mundo. Tamaño y distribución de la muestra: 81 unidades informativas (43 de Clarín y 38 de La Nación). En tanto el método de recogida de información incluye: a) lectura completa de toda 
la sección El Mundo de las ediciones que integran el lapso temporal bajo estudio; b) codificación correspondiente mediante plan estructurado y precodificado.

\section{Libro de códigos y variables}

Los encuadres noticiosos son variables latentes no observables ni medibles directamente sino que emergen a partir de una serie de indicadores manifiestos (IGARTÚA; MUÑIZ; CHENG, 2005, p. 157; MERCADO SAÉZ, 2013, p. 245). Para que la actividad sea operativa se requiere la construcción de un libro de códigos. Se trata de una herramienta que "define la forma en que los elementos de la realidad van a ser recuperados" (ACOSTA VALVERDE; PARRA ROSALES, 1995, p. 7) por lo que se deben definir variables descriptivas o formales e identificar indicadores manifiestos que se aplicarán a cada unidad de análisis. A través de las variables será posible brindar información de identificación de cada texto, grado de importancia, actores relevantes y categorías temáticas (IGARTÚA, 2006; MERCADO SAÉZ, 2013, p. 246).

Con esos indicadores, que serán codificados estableciéndose su presencia o ausencia en las unidades de análisis, será factible medir la prevalencia de los encuadres. Hemos definido, entonces, una serie de indicadores, siguiendo la propuesta de Igartúa et al. (2005):

1) Datos de identificación básicos: para cada unidad de análisis se identifica el diario, el día y mes de publicación y género de la publicación (sólo se analizan crónicas).

2) Emplazamiento de la información: si la información viene (1) o no de la portada (o); si abre (1) o no la sección (o); tamaño de la unidad de análisis (1: un cuarto de página, 2: tres cuarto de página; 3: mitad de página; 4: pagina completa); presencia (1) o no (o) de elementos gráficos como fotografías, gráficos o infografías; origen de la información: propia (corresponsal o enviado especial) (1) o de agencia de noticias (o). Sobre estas variables identificadas se realizó un análisis factorial de componentes principales (con rotación ortogonal) que identificó dos factores. El primer factor compuesto por las variables relacionadas con el tamaño de la información en el diario, la presencia de elementos gráficos, la apertura de la sección del diario y la aparición en portada. El segundo factor compuesto por una única variable: origen de la información. A partir de aquí se creó un índice de relevancia donde la información de máxima importancia sería aquella que apareciera en portada (1), abriera la sección del diario (1), incorporara algún tipo de información gráfica (1), el origen de la información fuera propia (1), se emplazara en página impar (1) y ocupara entre mitad de página a completa dentro del diario (1). El rango de variación de esta variable es: o-1 (relevancia 
mínima), 2-3 (relevancia media-baja), 4-5 (relevancia media), 6-7 (relevancia mediaalta) y 8-10 (relevancia máxima).

3) Encuadre de campaña: estratégico o temático (FLOSS; MARCINKOWSKI, 2008). Aquí definimos cuatro reactivos, medidos en presencia (1) o no presencia (o): 1) para el encuadre estratégico: a- el discurso enfatiza datos de sondeos de opinión; b-el discurso apela a expresiones relacionadas con la competencia entre candidatos; 2) para el encuadre temático: a- el discurso se centra en las propuestas de los candidatos; b- el discurso se centra en las problemáticas políticas, sociales y económicas del país (MUÑIZ; ROMERO, 2012).

4) Titulares: desde $A T L A S / t i$ importamos todas las unidades informativas. Asociamos a los títulos un conjunto de códigos que se desprendieron de su recurrencia en las unidades informativas. Este análisis cualitativo nos permitió dar cuenta de los encuadres cognitivos que se activaron a partir de la mecánica de titulación.

\section{Fiabilidad}

Una vez que la muestra fue codificada por dos codificadores previamente entrenados hicimos una tabla de datos en Excel que nos permitió realizar el análisis estadístico. Los gráficos fueron realizados con Tábleau.

Para garantizar la fiabilidad del proceso de codificación aplicamos el coeficiente de Kappa de Cohen. Los jueces codificaron un 20\% de la muestra seleccionada aleatoriamente y se obtuvo un índice de .83; es decir, un nivel de concordancia muy bueno.

\section{Resultados}

\section{Cobertura}

La prensa de referencia argentina publicó un total de 81 unidades informativas sobre el balotaje en Brasil con un promedio de 1.86 noticias por día para Clarín y 1.72 para La Nación. El siguiente gráfico muestra la distribución de las crónicas en el período de análisis: 


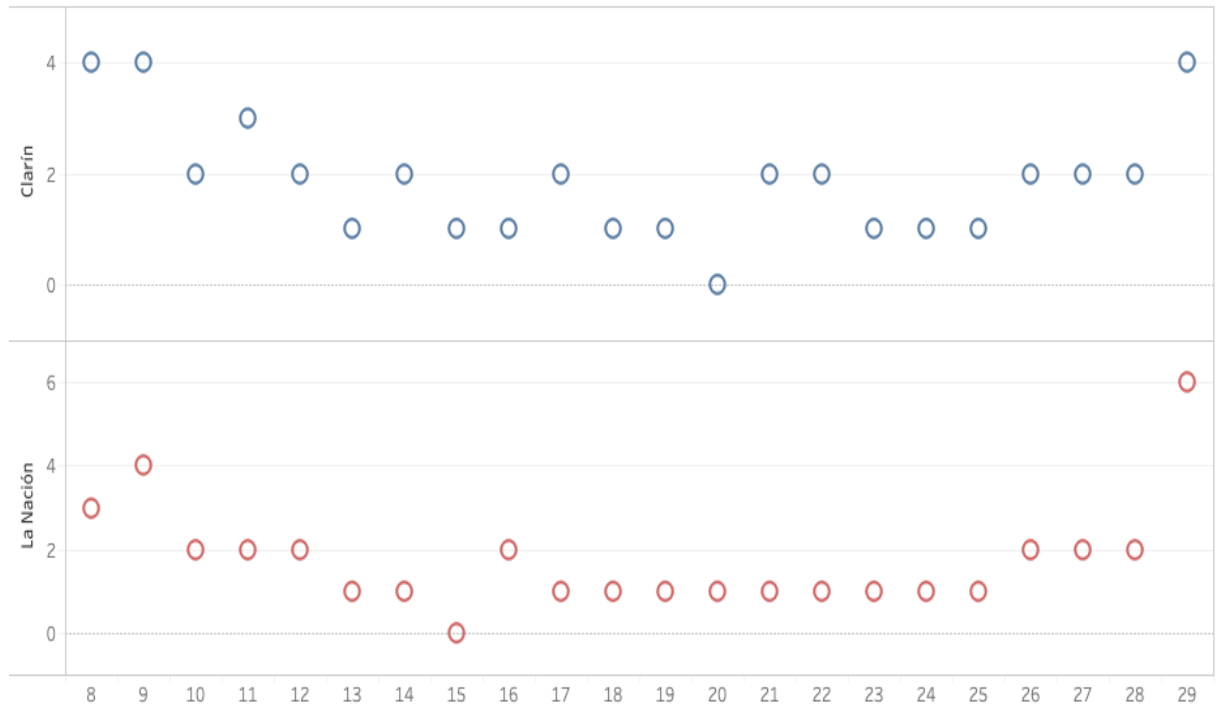

Figura 1: Distribución de unidades informativas

Fuente: Elaboración propia (2019).

La información sobre la segunda vuelta se mantuvo siempre presente excepto los días 15 y 20 de diciembre donde no se han registrado unidades informativas. Los picos informativos se observan el 8 y 9 de marzo tras el triunfo de Bolsonaro en la primera vuelta y el 29 de octubre tras los resultados del balotaje.

Respecto del emplazamiento de la información encontramos similitudes: 1) las noticias sobre los comicios fueron portada en 8 oportunidades en los dos medios y 11 veces abrieron las secciones El Mundo; 2) ambos ubicaron la información preferentemente en las páginas pares (63\% Clarín y 64.10\% La Nación).

Dentro de las diferencias destacamos que: 1) Clarín incluyó más elementos gráficos en sus unidades (79\%) que La Nación (59.97\%); 2) Clarín distribuyó las noticias en páginas enteras (31.81\%) y 3/4 de página (27.27\%); en tanto en La Nación predominaron los tamaños mitad de página (33.33\%) y página entera (30.76\%).

\section{Agenda temática de la sección}

La información del proceso electoral brasileño compartió la agenda de las secciones El Mundo con otros temas. En la Tabla 1 se observa la distribución temática y sus correspondientes porcentajes:

\begin{tabular}{|l|c|c|c|c|}
\hline \multicolumn{1}{|c|}{ Tema } & Clarín & La Nación & Total & $\%$ \\
\hline $\begin{array}{l}\text { Elecciones en } \\
\text { Brasil }\end{array}$ & 43 & 38 & 81 & 40.70 \\
\hline $\begin{array}{l}\text { Asesinato de } \\
\text { Khashoggi }\end{array}$ & 22 & 17 & 39 & 19.59 \\
\hline
\end{tabular}




\begin{tabular}{|l|c|c|c|c|}
\hline $\begin{array}{l}\text { Caravana de } \\
\text { migrantes a } \\
\text { EE.UU }\end{array}$ & 9 & 11 & 20 & 10.05 \\
\hline $\begin{array}{l}\text { Política interna } \\
\text { estadounidense }\end{array}$ & 3 & 5 & 8 & 4.02 \\
\hline $\begin{array}{l}\text { Política interna/ } \\
\text { externa china }\end{array}$ & 3 & 4 & 7 & 3.51 \\
\hline Brexit & 7 & 4 & 11 & 5.52 \\
\hline $\begin{array}{l}\text { Italia vs. UE } \\
\text { Casos de abusos } \\
\text { en la Iglesia }\end{array}$ & 5 & 4 & 9 & 4.52 \\
\hline $\begin{array}{l}\text { Tensión } \\
\text { comercial } \\
\text { EE.UU-China }\end{array}$ & 5 & 3 & 6 & 3.01 \\
\hline Venezuela & 4 & 3 & 6 & 4.02 \\
\hline $\begin{array}{l}\text { Detención de } \\
\text { Keiko Fujimori }\end{array}$ & 2 & 2 & 4 & 3.01 \\
\hline Total & 106 & 93 & 199 & 100 \\
\hline
\end{tabular}

Tabla 1: Agenda temática de sección El Mundo

Fuente: Elaboración propia (2019).

Durante el período estudiado, las elecciones en Brasil se han destacado (40.70\%) por sobre el resto de los temas agendados en la sección de información internacional. Le siguen en orden de importancia: el asesinato del periodista saudí Jamal Khashoggi en la embajada de Arabia Saudita en Estambul (19.59\%), la caravana de migrantes centroamericanos hacia la frontera de Estados Unidos (10.05\%) y el proceso de separación del Reino Unido de la Unión Europea (5.52\%).

El resto de los temas que completan el temario son: el conflicto entre Italia y la Unión Europea por el estancamiento económico del país (4.52\%), diferentes noticias sobre la política interna estadounidense (4.2\%), la "guerra comercial" entre Estados Unidos y China (4.02\%), la política interna/externa china (3.51\%), los casos de abusos en la Iglesia Católica (3.01\%), la "crisis venezolana" (3.01\%) y la detención por lavado de dinero de Keiko Fujimori en Perú (2.01\%).

\section{Indice de relevancia}

La siguiente tabla muestra el índice de relevancia que alcanzaron las unidades informativas sobre el proceso electoral brasilero [4]: 


\begin{tabular}{|c|c|c|c|}
\hline Relevancia & Clarín & La Nación & $\%$ \\
\hline $\mathrm{O}$ & $\begin{array}{c}1 \\
(2.27) \\
\end{array}$ & $\begin{array}{c}1 \\
(2.56)\end{array}$ & $2.4 \mathrm{O}$ \\
\hline 1 & $\begin{array}{c}3 \\
(6.81)\end{array}$ & $\begin{array}{c}\mathrm{O} \\
\text { (o) }\end{array}$ & 3.61 \\
\hline 2 & $\begin{array}{c}0 \\
(0)\end{array}$ & $\begin{array}{c}\mathrm{O} \\
(\mathrm{O}) \\
\end{array}$ & $\mathrm{O}$ \\
\hline 3 & $\begin{array}{c}1 \\
(2.27)\end{array}$ & $\begin{array}{c}\mathrm{O} \\
\text { (O) }\end{array}$ & 1.20 \\
\hline 4 & $\begin{array}{c}7 \\
(15.90)\end{array}$ & $\begin{array}{c}7 \\
(17.94)\end{array}$ & 16.83 \\
\hline 5 & $\begin{array}{c}2 \\
(4.54) \\
\end{array}$ & $\begin{array}{c}3 \\
(7.69) \\
\end{array}$ & 6.02 \\
\hline 6 & $\begin{array}{c}5 \\
(11.36) \\
\end{array}$ & $\begin{array}{c}6 \\
(15.38) \\
\end{array}$ & 13.25 \\
\hline 7 & $\begin{array}{c}3 \\
(6.81) \\
\end{array}$ & $\begin{array}{c}8 \\
(20.51) \\
\end{array}$ & 13.25 \\
\hline 8 & $\begin{array}{c}13 \\
(29.54) \\
\end{array}$ & $\begin{array}{c}6 \\
(15.38) \\
\end{array}$ & 22.89 \\
\hline 9 & $\begin{array}{c}5 \\
(11.36) \\
\end{array}$ & $\begin{array}{c}7 \\
(17.94) \\
\end{array}$ & 14.45 \\
\hline 10 & $\begin{array}{c}4 \\
(9.09)\end{array}$ & $\begin{array}{c}1 \\
(2.56)\end{array}$ & 6.02 \\
\hline Total & 44 & 39 & 100 \\
\hline
\end{tabular}

Tabla 2: Índice de relevancia

Fonte: Elaboración propia (2019).

Del análisis empírico resulta que las unidades que lograron el pico de importancia fueron aquellas que consiguieron 8 puntos (22.89\%); le siguen las que se ubican en los 6 y los 7 puntos con iguales porcentajes (juntas suman el 26.5\%). Así, se puede afirmar que la relevancia de las informaciones sobre las elecciones ha oscilado entre el rango medio-alto y máximo; es decir, se trata de informaciones que, en gran parte, reunieron los componentes que definen dicho índice: aparecen en portada, abren la sección del diario, incorporan algún tipo de información gráfica, el origen de la información es propia, se emplazan en páginas impares y ocupan entre mitad de página a completa dentro del periódico.

Mientras vemos que Clarín ha ubicado a sus crónicas en los 8 puntos (29.54\%), La Nación lo hizo en los 7 puntos (20.51\%). Para el primer periódico las noticias del proceso electoral brasileño han alcanzado el rango máximo de noticiabilidad; para el segundo, la relevancia se ubica en el rango medio-alto. 


\section{Temario electoral}

El temario de la prensa de referencia sobre el proceso electoral brasileño se concentró en las noticias que tenían como protagonista a Bolsonaro y en las que hemos denominado "otras informaciones" (83.94\%). Hadad quedó relegado de la tematización, tal como refleja la Tabla 3:

\begin{tabular}{|l|c|c|c|c|}
\hline \multicolumn{1}{|c|}{ Tema } & Clarín & La Nación & Total & $\%$ \\
\hline Bolsonaro & 18 & 14 & 32 & 39.50 \\
\hline Haddad & 5 & 8 & 13 & 16.04 \\
\hline $\begin{array}{l}\text { Otras } \\
\text { informaciones }\end{array}$ & 20 & 16 & 36 & 44.44 \\
\hline Total & 43 & 38 & 81 & 100 \\
\hline
\end{tabular}

Tabla 3: Temario

Fuente: Elaboración propia (2019).

Dentro del universo de noticias que conforman el ítem "Otras informaciones" (44.44\%) se encuentran resultados de sondeos, la investigación por la campaña de fakenews que se le atribuye a su partido, el clima de violencia política que vive el país, la incertidumbre por la política económica que llevará adelante el candidato de la ultraderecha y la situación "crítica" en la que queda el PT tras el triunfo de Bolsonaro en la primera vuelta y su triunfo en el balotaje.

Las noticias que tienen a Bolsonaro como epicentro (39.50\%) se enfocan en el porcentaje de votos que ha obtenido en ambas elecciones, su futuro gabinete, su plan para privatizar empresas públicas, sus políticas medioambientales, la futura relación con Estados Unidos, la Unión Europea y el Mercosur y su "cambio de estrategia" para conseguir nuevos votantes de cara a la segunda vuelta.

Las informaciones relacionadas con Haddad (16.04\%) reparan en el "cambio" de su discurso tras las elecciones de primera vuelta y sus estrategias para conseguir votos y las divisiones al interior de su partido.

\section{Origen de la información}

Uno de los elementos claves para determinar la importancia que un medio le da a una información internacional es su origen: sus enviados especiales, corresponsales o agencias de noticias. En la Figura 2 vemos cuál ha sido el origen de la información en uno y otro medio: 


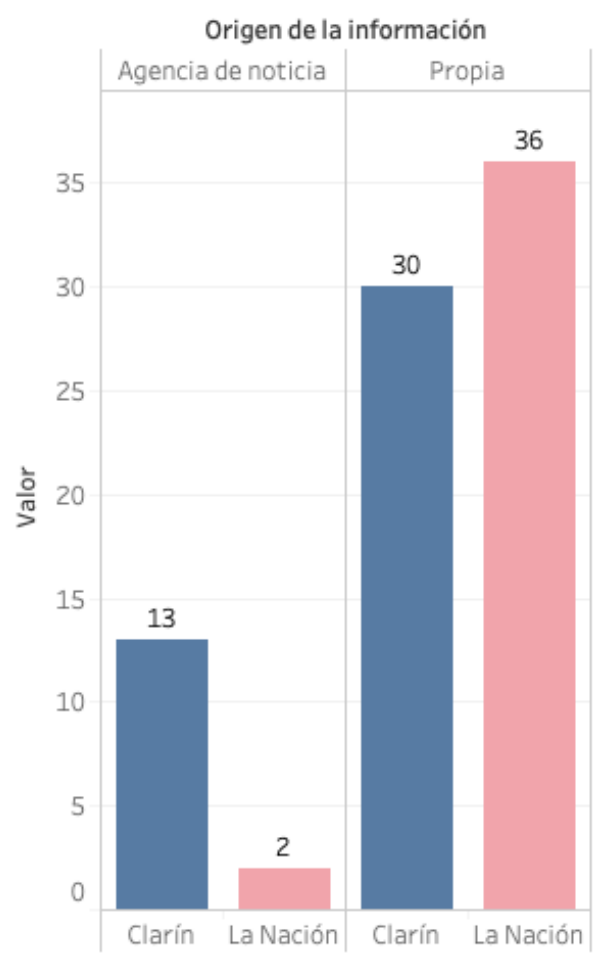

Gráfico 2: Origen de la información

Fuente: Elaboración propia (2019).

Ambos periódicos han utilizado información propia en la construcción discursiva de los comicios lo que muestra la importancia que ambos medios le han dado al proceso electoral brasileño. No obstante, casi toda la información de La Nación proviene exclusivamente de su enviado especial (9\%); en Clarín, el porcentaje es más bajo (69.76\%).

Todas las agencias de noticias utilizadas por la prensa de referencia han sido europeas: Agence France-Presse (AFP), AssociatedPress (AP), Deutsche PresseAgentur (DPA) y EFE.

\section{Encuadres}

Cuando setratade noticias internacionales, el públiconoconocenecesariamente a los actores que participan de los acontecimientos noticiables ni su contexto de actuación. En este sentido, el rol pedagógico del periodista en el tratamiento del tema se vuelve fundamental. Aún así, en el caso de las elecciones en Brasil, el encuadre de campaña que se impuso fue el estratégico (70.37\%). El uso del encuadre temático, consecuentemente, ha quedado relegado (29.62\%). La Figura 3 muestra la distribución de estos en cada medio: 


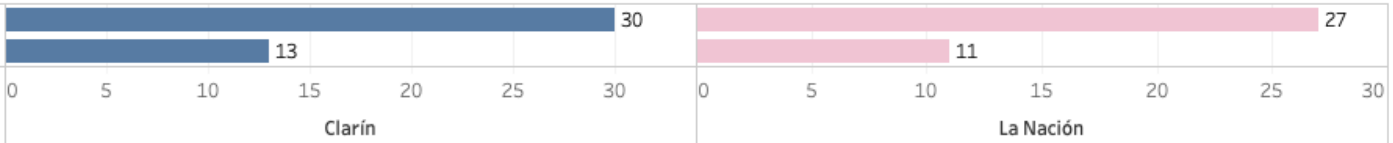

Gráfico 3: Encuadres

Fuente: Elaboración propia (2019).

Dentro del encuadre estratégico en ambos medios se destacan las expresiones de competencia (63.15\%) por sobre la publicación de resultados de sondeos de opinión $(36.84 \%)$, reduciendo el proceso electoral a una mera competencia entre candidatos.

\section{Titulares}

Una de las maneras de identificar el encuadre es a través del análisis de los titulares. La función de éstos es clave pues no son sólo señaladores que controlan la atención y la percepción del lector sino, además, su lectura lleva a que interprete el texto y forme nuevas opiniones o active las prexistentes.

El software ATLAS/ti nos ha permitido relacionar un conjunto de palabras clave (códigos) para dar cuenta de los encuadres cognitivos que la prensa de referencia ha activado sobre el proceso electoral a partir de su mecánica de titulación:

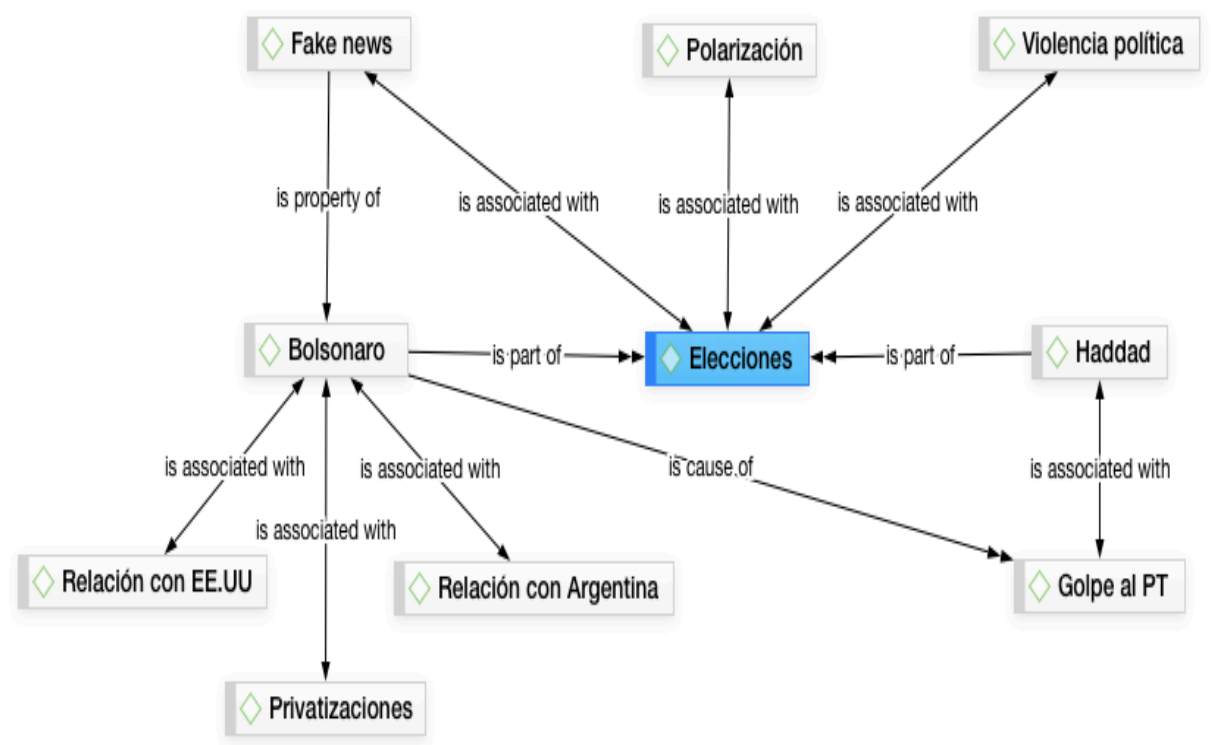

Gráfico 4: Red cognitiva

Fuente: Elaboración propia (2019)

Los medios han relacionado al proceso electoral brasilero con los conceptos de: polarización tras los resultados de las elecciones de la primera vuelta y los pronósticos para el balotaje; violencia política atribuida a los grupos ultraconservadores que siguen al candidato Bolsonaro y la campaña de fakenews a la que atribuyen al líder conservador por similitudes con la desarrollada por Donald Trump durante 2016. 
Bolsonaro concentra el interés de la prensa argentina, como hemos señalado en otro de los apartados. Puntualmente indagan en la relación que el nuevo presidente mantendrá con Estados Unidos y se muestran expectantes por la política externa que llevará adelante con la administración de Mauricio Macri. También hay incertidumbre sobre las potenciales privatizaciones de empresas estatales.

Leen la derrota de Haddad como la causa que desencadena una crisis al interior del PT. También agendan las críticas y discrepancias de sus socios Partido Democrático Laborista (PDT). El apoyo de los laboristas se presenta como fundamental para intentar revertir la enorme distancia de Haddad con respecto a Bolsonaro.

\section{Discusión y conclusiones}

Luego de presentar los resultados empíricos del análisis de la cobertura del proceso electoral brasilero por parte de la prensa de referencia argentina podemos realizar las siguientes conclusiones.

La prensa de referencia argentina ha prestado especial atención a las elecciones brasileñas. El índice de importancia de la cobertura ha oscilado entre el rango medioalto y máximo. Esto se corresponde con las posibles consecuencias que generaría para la relación bilateral la llegada de un gobierno cuya ideología ultraconservadora y de orientación derechista pudiese insuflar a Argentina.

Ambos medios han utilizado fuentes propias en la construcción del acontecimiento. Esto revela, como hemos demostrado, la importancia que dicha coyuntura electoral tuvo para los periódicos argentinos. Se ha evidenciado empíricamente que si bien ambos le dieron un similar nivel de cobertura, Clarín le dedicó apenas un número mayor de unidades informativas que La Nación. Asimismo, se corrobora que los días poseleccionarios (tanto en primera como en segunda vuelta) logran los mayores picos informativos.

Con relación al temario electoral, el protagonismo que adquirió Bolsonaro fue muy alto. De esta manera, se refuerza la tesis que va en dirección de afirmar el proceso creciente de personalización de la política, o mejor dicho de los atributos particulares del candidato que logró atrapar la atención del lector.

El encuadre predominante ha sido el estratégico (CAPIELLA; JAMESON, 2001; ALTHEIDE, 1973; ARTERTON, 1987), en sintonía con la mayoría de los estudios que señalan que en la cobertura de procesos electorales prima este tipo de frame, reduciendo las elecciones a una mera confrontación o competencia entre candidatos. El uso de este tipo de encuadre conlleva, en este caso, un riesgo particular. En pocas 
ocasiones como en las noticias internacionales, los destinatarios se encuentran tan vulnerables al mensaje periodístico. En este sentido, que un proceso electoral se presente como una simple carrera hacia la Presidencia de un país olvida la idea de que es el periodismo el que debe contextualizar la información que rodea al hecho noticioso (ALESSANDRI, 2012).

Sobre el punto anterior conviene advertir, además, que el estudio del framing de campaña electoral apenas comienza a ser abordado en la literatura argentina por lo que se presentan enormes posibilidades para la apertura y avance de un novedoso campo de estudio en las investigaciones empíricas de la comunicación política. Y, especialmente, existe una vacancia en el estudio acerca de las miradas que los productores de opinión ofrecen sobre acontecimientos de agenda política regional que aquí presentamos.

Los hallazgos de este estudio nos han permitido, por un lado, dar cuenta de las estrategias editoriales llevadas adelante por la prensa para tematizar el proceso electoral brasileño y, por otro, observar los flujos de opinión que se deprenden de su agenda con los consecuentes efectos cognitivos en el público (IGARTÚA et al., 2007; SAPERAS, 1987). Si tenemos en cuenta, en términos luhmannianos, que la tematización mediática funciona como guía de la opinión pública con nuestro trabajo hemos intentado indagar en los modos en que la prensa argentina ha acercado al público su construcción discursiva sobre la realidad brasileña.

\section{Notas}

[1] Fuente: Instituto Verificador de Circulaciones (IVC), Boletín Xpress, junio de 2019. Consulta: agosto de 2019.

[2] Fuente:https://www.clarin.com/sociedad/edicion-digital-clarin-supero-ano-10o-ooosuscriptores_o_HJbrCGtpM.html

[3] Para una mayor comprensión del periódico Clarín, ver Llonto (2003), Ramos (1993), Sivak (2013).

[4] Para calcular el índice de relevancia tuvimos en cuenta las dos veces que ambos medios no publicaron información sobre las elecciones en Brasil. A cada una de ellas las ubicamos en o y le asignamos un 1 por cada medio, tal como se ve en la Tabla 2.

\section{Referências}

ACOSTA VALVERDE, M.; PARRA ROSALES, L. Los procesos electorales en los medios de comunicación: guía para el análisis electoral en México. México: Universidad Iberoamericana, 1995.

ALESSANDRI, F. Periodismo Internacional: la ruptura de fronteras. Cuadernos.Info, V. 8, P. 
ALTHEIDE, D. Creating Reality: How TV News Distorts Events. Londres: Sage, 1973.

AMADEO, B. La teoría del Framing. Los medios de comunicación y la transmisión de significados. Revista de Comunicación, v.1, p.6-32. 2002. https://revistadecomunicacion.com/ es/articulos/2002/Artoo6-32.html. Consulta: agosto 2019.

ARTERTON, C. Las estrategias informativas de las campañas presidenciales. México: Publigrafic, 1987.

ARUGUETE, N. El poder de la agenda. Política, medios y público. Buenos Aires: Biblos, 2015.

BERGANZA CONDE, M. R. Medios de comunicación, ‘espiral del cinismo’ y desconfianza política. Estudio de caso de la cobertura mediática de los comicios electorales europeos. Revista Zer, .v. 13, n.25, p.121-139. 2008. https://www.ehu.eus/ojs/index.php/Zer/article/ view/3580. Consulta: agosto 2019.

BORRAT, H. El periódico, actor político. Barcelona: GG Mass media, 1989.

BORRAT, H.; FONTCUBERTA, M. Periódicos: sistemas complejos, narradores en interacción. Buenos Aires: La Crujía, 2006.

CAPELLA, J.; JAMIESON, K. H. Spiral of cynicism: the press and the public good. Oxford: Oxford University Press, 1997.

GASERMEIRO, A. Los medios en las elecciones. La Agenda Setting en la Ciudad de Buenos Aires. Buenos Aires: Educa, 2004.

DE VREESE, G. H., BOOMGAARDEN, H. G.; SEMETKO, H. A. (In)direct Framing Effects: The Effects of News Media Framing on Public Support for Turkish Membership in the European Union. Communication Research, .v. 38, n. 2, p. 179-200. 2011. https://journals.sagepub.com/ doi/10.1177/0093650210384934. Consulta: agosto 2019.

DÍAZ, G. Diario de guerra. Clarín el gran engaño argentino. Buenos Aires: Ediciones de la V, 2009.

FLOSS, D.; MARCINKOWSKI, F. Do Media News Frames Reflect a Nation's Political Culture? (Working Paper n 25). Zurich: National Centre of Competence in Research. 2008.

GAGLIARDI, J. La elección de 2018 y la gran prensa brasileña. Revista Politica Latinoamericana, v.7, 1-9, 2018.

GROSSI, G. Rappresentanza e reppresentazione. Milano: Franco Angeli, 1985.

HIRST, M. Argentina-Brasil: Perspectivas comparativas y ejes de integración. Buenos Aires: Editorial Tesis, 1990.

IGARTÚA, J.J.; MUÑIZ, G.; OTERO, J.A.; FUENTE J.M. El tratamiento informativo de la inmigración en los medios de comunicación españoles. Un análisis de contenido desde la Teoría del Framing. Estudios sobre el Mensaje Periodístico, v.13, p.91110. 2007. https://revistas. ucm.es/index.php/ESMP/article/view/ESMPo707110091A. Consulta: agosto 2019.

IGARTUA, J. J., MUÑIZ, G. Y CHENG, L. La inmigración en la prensa española. Aportaciones empíricas y metodológicas desde la teoría del encuadre noticioso. Migraciones, v.17, p.143181. 2005. https://revistas.comillas.edu/index.php/revistamigraciones/article/view/4220. 
Consulta: agosto 2019.

KEJSEFMAN, I. La inserción internacional y el conflicto distributivo en la dinámica del ciclo de acumulación en América Latina: los casos de Argentina y Brasil [2001-2011] Tesis de Maestría(UBA), 2017.

KRIPPENDORFF, K. Metodología de análisis de contenido. Teoría y práctica. Barcelona: Paidós, 1990.

LLONTO, P. La noble Ernestina. Buenos Aires: Astralib, 2003.

LUHMANN, N. La realidad de los medios de masas. México: Universidad Iberoamericana, 2000.

MARLETTI, C. Prima e dopo. Tematizzazione e comunicazionepolitica. Turin: Rai/Vpt, 1985.

MAURENTE LAFLOR, L. "Elecciones presidenciales de 2010 en Brasil: como la prensa argentina habla de los candidatos". Tesis de Maestría (UBA), 2016.

MCCOMBS, M.; SHAW, D. The agenda-setting function of the mass media. Public Opinion Quarterly, v.36, p.176-187, 1972. https://www.jstor.org/stable/2747787?seq=1\#page_scan_tab_ contents. Consulta: agosto 2019.

MERCADO SAEZ, M. Diseño metodológico para el análisis del tratamiento informativo de las políticas energéticas en España y participación de organizaciones sociales. Ponencia presentada en el Congreso Nacional sobre Metodología de la Investigación en Comunicación. Segovia. 2013.

MUÑIZ, D.; ROMERO, A. Framing de la campaña electoral mexicana. Análisis de los encuadres noticiosos sobre política presentes en los principales diarios digitales mexicanos de ámbito nacional.Actas -IV Congreso Internacional Latina de Comunicación Social- IV CILCS - Universidad de La Laguna. [no prelo], 2012.

MUÑIZ, D.; SALDIEMA, E.; DE JESÚS, F. The Stages of the Campaign as a Moderator of the Presence of Political Frames in the News. Palabra Clave, v.21, n. 3, p. 740-771. 2018. http:// www.scielo.org.co/pdf/pacla/v21n3/0122-8285-pacla-21-03-00740.pdf. Consulta: agosto 2019.

PATTERSON, T. Out of Order. Nueva York: Alfred A. Knopf, 1993.

RAMOS, J. Los cerrojos de la prensa. Buenos Aires: Editorial Amfin, 1993

SÁDABA, T. Framing: el encuadre de las noticias. El binomio terrorismo-medios. Buenos Aires: La Crujía, 2008.

SANTILLÁN, J.R. La prensa mexicana ante el cambio político. Madrid: Ed. Dykinson, 2009.

SAUTU, R. Todo es teoría: objetivos y métodos de investigación. Buenos Aires: Lumiere, 2005. RODRIGO ALSINA, M. La construcción de la noticia. Barcelona: Paidós, 1989.

RUSSELL, R.; TOKATLIAN, J. El lugar de Brasil en la política exterior argentina. Buenos Aires: Fondo de Cultura Económica, 2003.

SAPERAS, E. Los efectos cognitivos de la comunicación de masas. Las recientes investigaciones en torno a los efectos de la comunicación de masas, 1970-1986. Madrid: Ariel, 1987. 
SIDICARO, R. La política mirada desde arriba. Las ideas del diario La Nación 1909-1989. Buenos Aires: Sudamericana, 1993.

SIVAK, M. Clarín. El gran diario argentino. Una historia. Buenos Aires: Planeta, 2013.

STEIMBERG, O.; TRAVERSA, O. Estilo de época y comunicación mediática. "Por donde el ojo llega al diario: el estilo de la primera página”. Buenos Aires: Colección del círculo, 1996.

TANKARD, J.; HENDRICKSON, L.; SILBERMAN, J., BLISS, K.; GHANEM, S.: "Media Frames: Approaches to Conceptualization and Measurement”. Ponencia presentada en Comnunication Theory and Methodology Division. AEJMC Convention, Boston, 1991.

ULANOVSKY, G. Paren las rotativas (1920-1960).Buenos Aires: Emecé, 2005. 